\title{
Modelo de Dinámica de Sistemas para la Predicción del Comportamiento del Mercado Porcícola
}

\author{
Yony F. Ceballos ${ }^{(1)}$, Maribel Uribe ${ }^{(2)}$ y German Sánchez ${ }^{(3) \text { * }}$ \\ (1) Instituto Tecnológico Metropolitano, Programa de Ingeniería de Sistemas, Calle 73 No. 76A-354, \\ Medellín-Colombia (e-mail: fernandoceballos@itm.edu.co). \\ (2) Universidad de Antioquia, Facultad de Ingenierías, Programa de Ingeniería de Sistemas, \\ Calle 70 No. 52-21, Medellín-Colombia (e-mail: maribel.uribe@.udea.edu.co). \\ (3) Universidad del Magdalena, Facultad de Ingeniería, Programa de Ingeniería de Sistemas, \\ Grupo de Investigación y Desarrollo en Nuevas Tecnologías de la Información y la Comunicación, \\ Carrera 32 No. 22-08, Santa Marta - Colombia (e-mail: gsanchez@unimagdalena.edu.co).
}

* Autor a quien debe ser dirigida la correspondencia

Recibido Ene. 24, 2013; Aceptado Mar. 19, 2013; Versión final recibida Mar. 26, 2013

\begin{abstract}
Resumen
En este artículo se presenta la construcción de un modelo basado en dinámica de sistemas para describir el comportamiento del mercado porcícola. Esto con el objetivo de anticiparse a los posibles acontecimientos, crear escenarios supuestos y determinar si es viable o no invertir en el corto a mediano plazo en este mercado. El modelo propuesto se compone de siete ciclos que consideran las diferentes variables que intervienen en el medio, se establece las relaciones y se construyeron los ciclos entre éstas. Se configuraron las variables del modelo con datos históricos del sector. Los resultados muestran una gran dependencia del costo de la materia prima sobre las ganancias y establece el escenario de cría como el más rentable.
\end{abstract}

Palabras claves: pensamiento sistémico, porcicultura, simulación, dinámica de sistemas.

\section{System Dynamics Modeling for Pig Breeding Market Behavior Prediction}

\begin{abstract}
This paper presents the construction of a model based on system dynamics for describing the behavior of pig market. This with the objective of anticipating possible events, creating scenarios about assumptions and determine whether it is feasible or not to invest in this market in a short to medium term. The proposed model consists of seven cycles considering the different variables involved in the pig market, establishing relationships and the cycles formed between them. The model variables were set using historical data of the market. The results show a high dependence of raw material costs over the earnings and show the stage of breeding as the most profitable scenario.
\end{abstract}

Keywords: systems thinking, porciculture, simulation, system dynamics 


\section{INTRODUCCIÓN}

La explotación porcina representa un renglón importante en la economía agrícola colombiana, al ser un medio de producción alternativo de los cultivos tradicionales debido a que no se requiere de extensos territorios para su ejecución (Porcicol, 2009). Para el 2009 la población porcina se estimó en cerca de 4 millones de animales, de los cuales el $35 \%$ se concentro en Antioquia. Del total de esta industria, el 0,6\% estaban dedicados a reproducción, un $51,7 \%$ a engorde, el $6,5 \%$ a cria/recria y un $41,2 \%$ a lechón. Sin embargo sólo el 1,1\% corresponde a explotación tecnificada (FAO, 2009). Por tal razón, esta industria tiene un camino en dirección a la tecnificación y a la incorporación de tecnologías que permitan su desarrollo y crecimiento. En este sentido, al emprender una exploración en cualquier ámbito, es un aspecto importante reconocer las fortalezas y debilidades que presenta el medio. De una correcta comprensión de los diferentes componentes del mismo, sus relaciones y sus comportamientos, dependerá en gran parte el éxito o fracaso de la inversión, así como la sostenibilidad de la empresa en el tiempo.

Cuando existen limitaciones en la comprensión de los componentes y sus relaciones, los modelos de simulación constituyen una herramienta esencial para la toma de decisiones, debido a que permiten explicar el comportamiento, funcionamiento, características y las respuestas de los sistemas de producción a las diversas situaciones que suelen presentarse, permitiendo describir el problema, evaluar los efectos en búsqueda de soluciones reduciendo en gran medida las restricciones de tiempo y de costos (Robinson, 2004; Camargo y García, 2012). Dentro de los diferentes esquemas de simulación, la Dinámica de Sistemas constituye el esquema que toma el Pensamiento Sistémico como parte esencial en la toma de decisiones, lo que permitirá establecer criterios aproximados a la realidad, que determinen la tendencia de las diferentes variables involucradas en el medio, y de esta forma, tomar medidas que conlleven a lograr el mejor desempeño posible (Capelo y Dias, 2009).

En el caso particular del mercado porcícola, la heterogeneidad de actores y situaciones presentes en el medio conlleva a que el comportamiento del sistema sea complejo y confuso (Espinal et al., 2005). Tal situación, hace difícil la toma de decisiones a aquellas personas que se encuentren interesadas en la explotación de este campo y que deseen medir el impacto financiero que tendría su inversión a mediano y largo plazo de una manera rápida y eficiente. La falta de proyecciones, tendencias y control de las diferentes situaciones que se puedan presentar en el medio porcicultor, conduce a que la inversión de capital en este campo se realice con mucho temor y riesgo de pérdida, al no contarse con un mecanismo que permita visualizar el comportamiento y tendencias que tendrían las diferentes variables que intervienen en el sector (Espinal et al., 2009).

La incorporación de las teorías del Pensamiento Sistémico a la comprensión de las dinámicas de los mercados está demarcada por trabajos realizados por la comunidad científica del área, direccionados hacia el modelamiento de diferentes procesos en un grupo variado de mercados en el sector agrícola (Kunc, 2012). En este contexto, el sector porcino es considerado un área de estudios nueva y constituye un área de interés poco explorada (Espinal et al., 2009).

Diferentes ejemplos de la aplicación de la dinámica de sistemas en diferentes áreas se presentan en (Paich et al. 2011; Cuartas et al. 2004). Estos trabajos reportan herramientas informáticas en las cuales se describe la construcción de modelos matemáticos orientados a la realización de simulaciones de las relaciones y dinámicas de los diferentes elementos del sector ganadero. Otro ejemplo de aplicabilidad de la Dinámica de Sistemas en un mercado similar, lo encontramos en (Giraldo y Marín, 2004). Este trabajo se orienta al sector floricultor, en el cual se propone un modelo basado en la interacción de tres componentes principales. La Conceptualización, que enmarca los objetivos que conllevan a determinar la capacidad del medio, sus relaciones y sus límites. La segunda componente denominada Formulación del Modelo, tiene como fin, mediante la inversión en estudios sobre el comportamiento del medio y adelantos tecnológicos, reducir costos en procesos productivos para poder competir con precios bajos en un medio que se encuentra sobre ofertado. La última componente que se encuentra, se denomina Evaluación. En esta componente se determina qué porcentaje de reducción de costos se logra obtener en el proceso productivo por cada peso invertido en investigación y desarrollo creándose así, escenarios detectados para lograr mayor eficiencia en el ámbito comercial.

En el sector lácteo, una incursión de la Dinámica de Sistemas se presenta el trabajo de Cuartas et al. (2004), el cual describe una simulación realizada para comercialización de la leche líquida de corta duración. La simulación fue realizada a diferentes estrategias de comercialización y distribución, creando modelos en las variables de mercado como lo son precio, clientes, canales de distribución, publicidad, promoción y fuerza de ventas. Restrepo et al. (2005), describen un modelo realizado en el sector de las Pymes de productos cárnicos. Se muestran los problemas más frecuentes en el sector de la producción de cárnicos y se le plantean soluciones mediante la creación de tres diagramas causales que ilustran el comportamiento de estos inconvenientes en el sector y sus componentes 
El presente trabajo está orientado a la construcción de un modelo basad en Dinámica de Sistemas que permita una mejor comprensión de las variables y su relaciones dentro del mercado porcicola. Esto permitirá predecir la tendencia de las diferentes variables involucrados en la producción y comercialización de los cerdos a mediano plazo mediante la simulación y el análisis de resultados.

\section{MODELO PROPUESTO DEL MERCADO PORCICOLA}

El modelo propuesto se compone de siete ciclos que consideran las diferentes variables que intervienen en el medio. El diagrama causal de la figura 1, establece las relaciones entre las diferentes variables, los ciclos conformados entre ellas y la dinámica que se presenta. Para lograr establecer la dinámica actual existente en el medio, se encontró apoyo en el manual PIC (Pig Improvement Company) donde se ilustra la forma de ejercer porcicultura de manera tecnificada. Los datos tales como costos de producción, precios de comercialización de lechones y de cerdo en pie en las diferentes épocas fueron obtenidos del Fondo Nacional de Porcicultura (Porcicol, 2009).

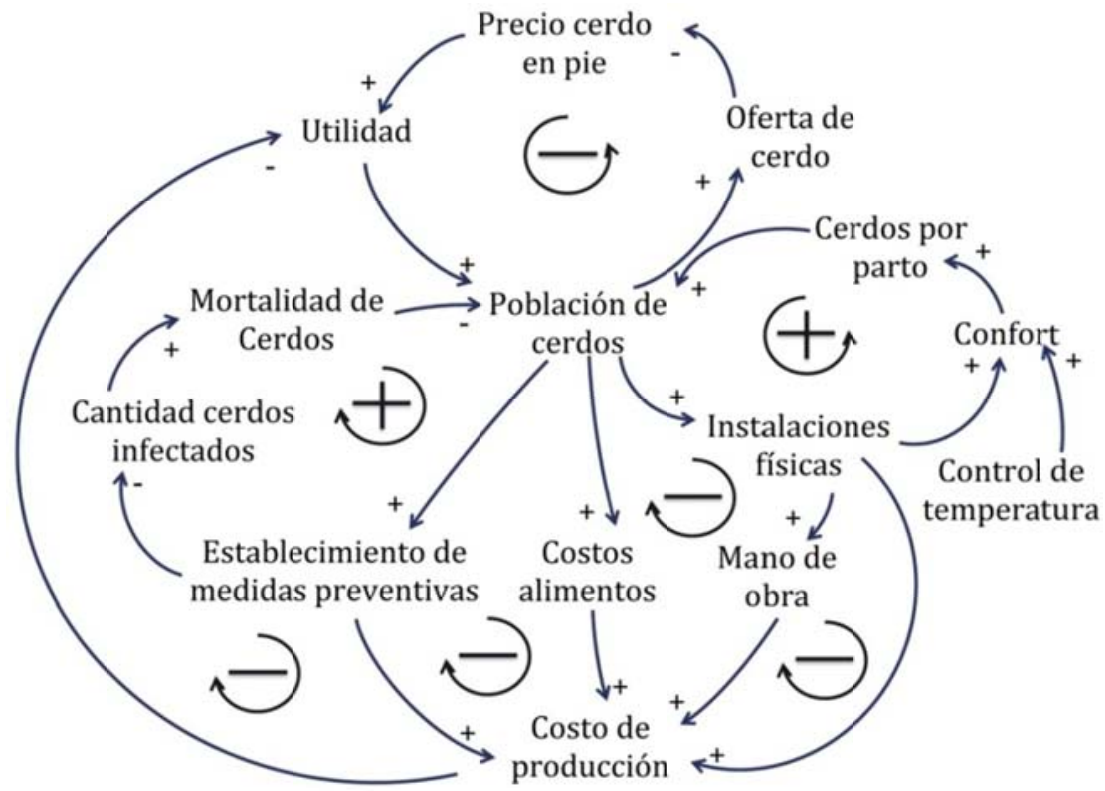

Fig. 1: Hipótesis dinámica del medio porcicola.

\section{Descripción de la hipótesis dinámica}

La oferta de cerdos es determinada por la población de cerdos, es decir, a mayor población de cerdos mayor será la oferta de ellos. La cantidad de cerdos (oferta) que se encuentra disponible para la comercialización (lechones y/o cerdos cebados), determinarán en gran medida el precio del cerdo, ya que a mayor oferta, menor será el precio. El precio de cerdo en pie es estimado de acuerdo con una serie de precios históricos (Porcicol, 2009), que permiten encontrar la tendencia de éste por medio de una regresión (ver figura 2a).

El ciclo de la figura $2 b$, permite estudiar el comportamiento de la tasa de mortalidad presente en cada una de las etapas de los cerdos, si se sigue un conjunto de normas sobre higiene y vacunación que se denominan medidas preventivas. Si se cumple el conjunto de estas medidas en caida etapa, la cantidad de cerdos infectados tendría una tasa estimada relacionada con la etapa en la que se encuentre. Esta tasa de cerdos infectados permite considerar la cantidad de cerdos que morirán en cada una de las etapas y visualizar el comportamiento que se presentaría en la población de cerdos.

En este mismo sentido, el ciclo de la figura 2c, permite establecer los costos causados por el establecimiento de medidas preventivas en la granja. Es claro que al existir mayor población de cerdos, el establecimiento de medidas preventivas se incrementa y por ende los costos. Este incremento en los costos de producción afecta la utilidad pero permite tener una población de cerdos mayores y con mejores calificaciones sanitarias para obtener certificaciones que conlleven a encontrar nuevos nichos en el exterior.

Las instalaciones físicas constituyen un factor de importancia sobre la utilidad y la población de cerdos. En la figura $2 d$, se establece la influencia de los costos causados por las instalaciones físicas. Entre más población de cerdos hay en la granja, mayor deben ser las instalaciones para poder albergarlos. El 
estimativo de los costos causados por las instalaciones físicas requeridas en el medio porcícola se estima mediante una regresión obtenida por medio de una serie de datos históricos (Porcicol, 2009), que permiten establecer la tendencia del precio en el tiempo en sus diferentes etapas y de acuerdo con la población de cerdos existentes en cada una de ellas. Teniendo en cuenta la cantidad de cerdos presentes en cada etapa, se realiza un estimativo de los costos involucrados por instalaciones físicas y se visualiza la influencia de los costos en la utilidad.

La Figura 2e muestra el ciclo de mano de obra. El costo estimado de la mano de obra se realiza sobre cada etapa y sobre cada cerdo. De acuerdo con la etapa en que se encuentre, el precio pagado por mano de obra es diferente; y este costo se le aplica a cada cerdo en cada etapa. La base conceptual de este ciclo es que al aumentar la población porcina, se debe aumentar la capacidad de instalación, y al aumentarla ésta, se requiere un mayor número de trabajadores para poder cubrir el área total. Este incremento de personal, incrementa los costos de producción y afecta la utilidad.

En relación a los costos de producción, la variable más importante la constituye el costo de los alimentos durante el periodo de tiempo de cría. Durante este periodo de tiempo el alimento en la explotación porcina corresponde al $75.4 \%$ de los costos totales. En este sentido, es importante anotar que los cerdos en sus diferentes etapas presentan un consumo característico. Este ciclo se muestra en la figura $2 \mathrm{f}$, se caracteriza por que al aumentar la población porcina, se aumenta el consumo de alimento y al aumentar éste se requerirá una mayor cantidad de alimento. Así, el incremento de alimento, incrementa los costos de producción. Un factor de relevancia del modelo lo constituye las condiciones del habitad y es modelado como se muestra en la figura $2 \mathrm{~g}$. Este ciclo nos muestra que si se cumple un rango de temperatura óptimo y las instalaciones físicas son las adecuadas, el número de cerdos por parto se incrementará en un 10\%, ya que el animal presenta un mayor confort, lográndose así, un aumento en la población porcina.

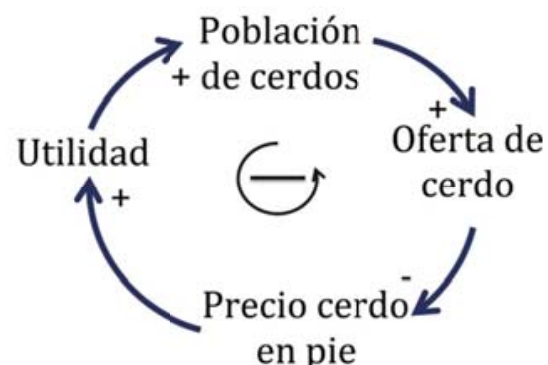

a)

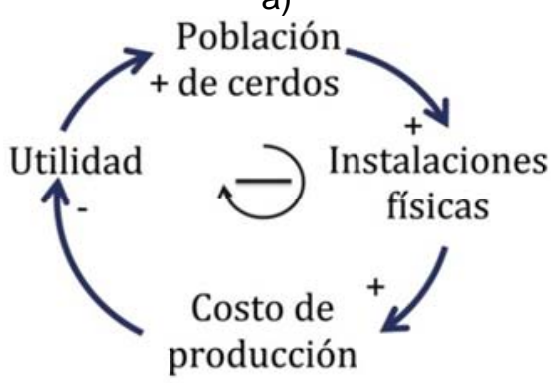

d)

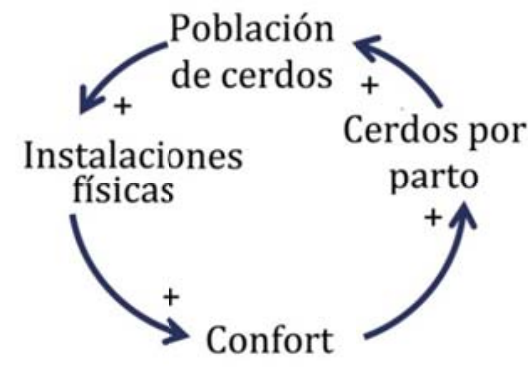

g)

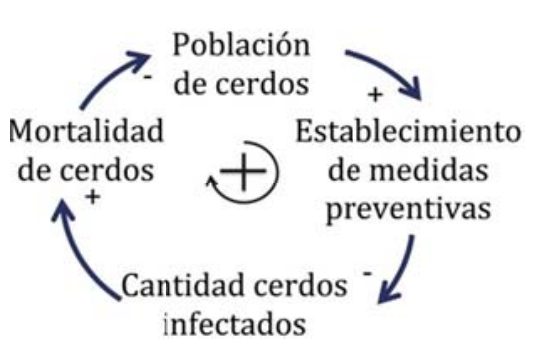

b)

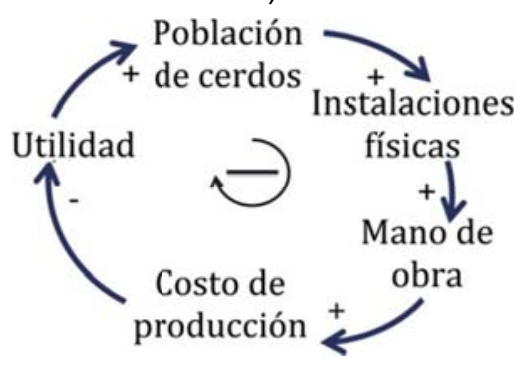

e)

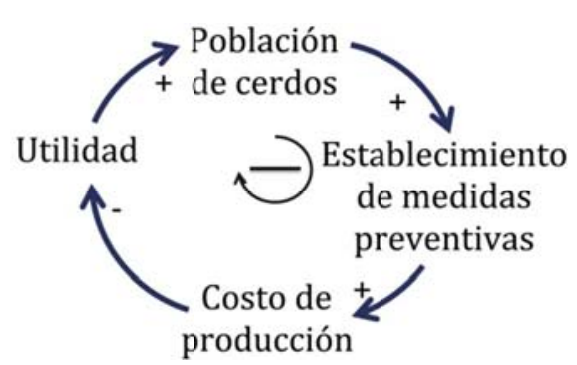

c)

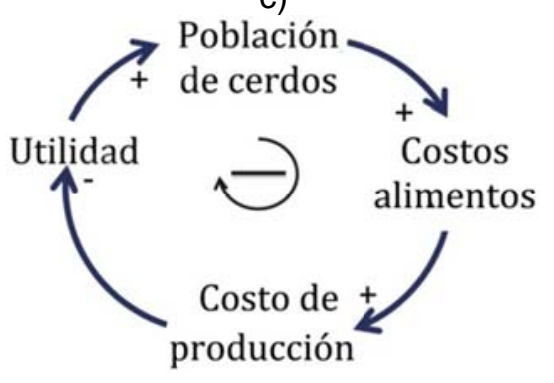

f)

Fig. 2: Diferentes ciclos que componen la hipótesis dinámica. a) Producción y comercialización. b) Establecimiento de las medidas preventivas. c) Costos del establecimiento de medidas preventivas. d) Costos de las instalaciones. e) Costos de la mano de obra. f) Costos de la alimentación. g) Condiciones de hábitat. 


\section{DATOS Y VALIDACIÓN DEL MODELO}

Para la caracterización del modelo se realiza a pasos de un día por una duración de 6 años. Los datos de entrada para las variables de cerdas para reproducción comienzan en 10 y alcanza un valor máximo de 200. Respecto a la cantidad de lechones por parto el valor es de 5 y a la cantidad de cerdos reproductores sólo es necesario tener en la finca uno. Los valores correspondientes a los costos de los alimentos, el costo de mantenimiento de la granja y a la utilidad percibida por la venta de cerdos, lechones y carne son tomados de Porcicol (2009); Espinal et al. (2005) y Padilla (2010).

Tabla 1. Variables y valores de entrada del modelo.

\begin{tabular}{|l|l|l|l|}
\hline \multicolumn{1}{|c|}{ Variable } & \multicolumn{1}{c|}{ Intercepto } & \multicolumn{1}{c|}{ Pendiente } & \multicolumn{1}{c|}{$\mathrm{R}^{2}$} \\
\hline Costo Alimento Lechón & 37902,122 & 90,423 & $0,311^{*}$ \\
\hline Costo Alimento Preceba & 25442,393 & 77,704 & $0,485^{*}$ \\
\hline Costo Alimento Ceba & 194009,263 & 33,920 & $0,003^{*}$ \\
\hline Costo Mano de Obra Lechón & 9189,609 & 50,527 & 0,821 \\
\hline Costo Mano de Obra Preceba & 2316,105 & 14,121 & 0,897 \\
\hline Costo Mano de Obra Ceba & 10220,956 & 54,144 & 0,881 \\
\hline Costo Medidas Preventivas Lechón & 2278,333 & 3,874 & $0,291^{*}$ \\
\hline Costo Medidas Preventivas Preceba & 3670,360 & 28,846 & 0,889 \\
\hline Costo Medidas Preventivas Ceba & 271,832 & 0,115 & 0,692 \\
\hline Costo Instalaciones Físicas Lechón & 3707,131 & 6,085 & $0,486^{*}$ \\
\hline Costo Instalaciones Físicas Preceba & 1924,210 & 13,559 & $0,410^{*}$ \\
\hline Costo Instalaciones Físicas Ceba & 2535,077 & 5,392 & 0,694 \\
\hline Precio Venta Lechón & 95772,901 & 1523,144 & 0,826 \\
\hline Precio Cerdo en Pie & 2514,426 & 44,756 & 0,7587 \\
\hline Variables de menor valor & & \\
\hline
\end{tabular}

Partiendo del análisis de las regresiones realizadas para calcular la tendencia de algunos costos en el tiempo se tiene que en las variables que contienen las series de costos de alimento en las diferentes etapas presentan un valor de ajuste $\mathrm{R}^{2}$ bajo (ver * en la tabla 3), lo cual indica irregularidad en los datos involucrados. Este fenómeno también se evidencia en la serie de costos de medidas preventivas para los lechones, la serie de instalaciones físicas para los lechones y cerdos en preceda. En las demás series realizadas los $R^{2}$ encontrados están en un rango alto, mayores a 0.65 , lo cual significa que la frontera tendencial arroja resultados de acuerdo con la realidad histórica planteada.

\section{RESULTADOS Y DISCUSIÓN}

Diferentes escenarios fueron considerados en relación a las cerdas reproductoras. La cantidad de las reproductoras se define en función de las dimensiones de la granja, las cuales presentan diferentes condiciones de espacio, temperatura y limpieza. La capacidad de la granja modelo utilizada para las simulaciones se estableció con una capacidad instalada máxima de 12 cerdas reproductoras. Esta consideración es importante debido a que son éstas las que inciden sobre la cantidad de lechones que se producirán, con repercusiones sobre la cantidad de unidades vendidas y cebadas en granja.

\section{Análisis de escenario tendencial}

Para el análisis tendencial se parte de una compra de 10 cerdas reproductoras y una venta de lechones acorde con la demanda típica del sector. La figura $3 a$, ilustra el comportamiento seguido por los ingresos y los costos de producción de acuerdo con la tendencia y a los parámetros iniciales establecidos para las cerdas reproductoras y lechones a vender. Se observa que la mayoría del tiempo los costos superan los ingresos. Esta situación se debe a la forma en la cual varía la utilidad, la cual está comprometida con los costos de producción (relacionados con el confort, la mano de obra y la adquisición de cerdas reproductoras). En este escenario además se observa que la utilidad es negativa, ya que los costos superan la los ingresos, volviendo este mercado poco atractivo para la inversión.

\section{Análisis Escenario 10 Madres Reproductoras Y Diferentes Porcentajes De Venta De Lechones}

Este escenario es planteado con la compra de 10 cerdas reproductoras mensuales y la venta de lechones en diferentes porcentajes. Se han planteado cinco escenarios adicionales en este contexto, a saber escenario reproductoras-lechon $\mathrm{N}$, para $\mathrm{N}=1, \ldots, 5$. En estos escenarios se compran diez madres reproductoras y se venden, 0, 25, 50, 75 y 100\%, respectivamente. El comportamiento de la utilidad se muestra en la figura 3b. Las utilidades en todos estos escenarios siempre hay una disminución del nivel de utilidades, pero hay recuperación al final de la simulación, lo cual da a entender que existe poca sensibilidad a esta variable en el modelo propuesto, y una mayor utilidad con la situación tendencial (línea superior en todos los gráficos posteriores). 


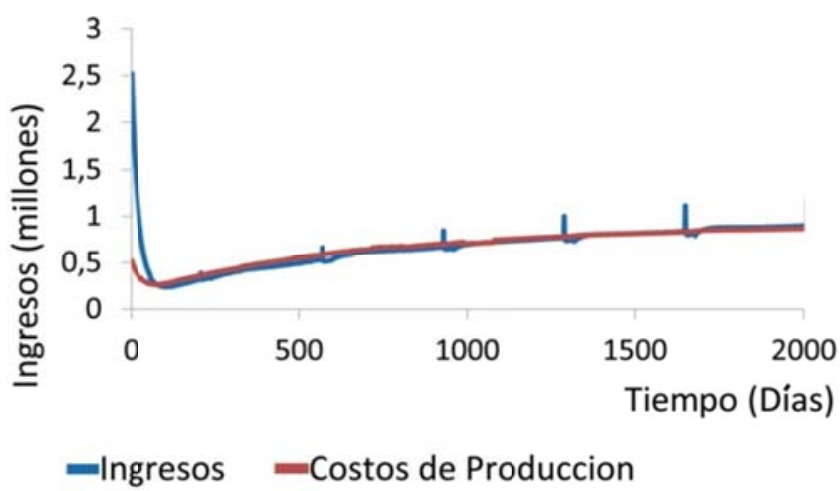

a)

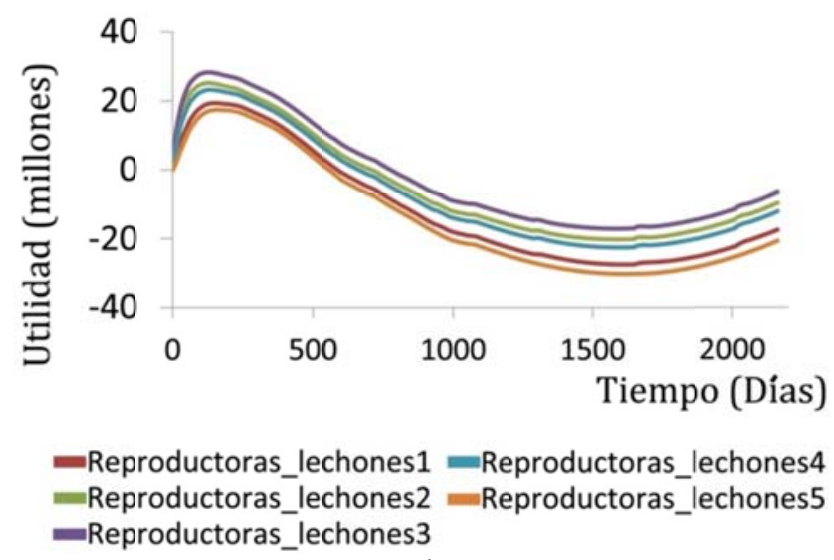

b)

Fig. 3: a) Comportamiento de los ingresos (en pesos Colombianos) y costos en el escenario tendencial, b) Escenario 10 madres reproductoras.

\section{Análisis Escenario 12 Madres Reproductoras Y Diferentes Porcentajes De Venta De Lechones}

De forma similar, se realizaron simulaciones en el escenario correspondiente a la compra de 12 madres reproductoras y la venta de lechones en diferentes porcentajes. Los escenarios reproductoras-lechón6, hasta reproductoras-lechón10, corresponden a la venta de 0, 25, 50, 75 y $100 \%$, respectivamente. El comportamiento de la utilidad se muestra en la figura 4a. Respecto al escenario tendencial, disminuyen los costos, dando señales de que la mejor forma de mejorar los ingresos a largo plazo en con una buena cantidad de cerdas reproductoras.

\section{Análisis Escenario 8 Madres Reproductoras Y Diferentes Porcentajes De Venta De Lechones}

El escenario reproductoras-lechón11, hasta el escenario reproductoras-lechon15, corresponde a la compra de 8 cerdas reproductoras mensuales y la no venta de lechones, es decir, cebar todos los lecho15, corresponden a los escenarios en los cuales se adquiere 8 madres reproductoras y se venden un porcentaje de lechones del $0 \%, 25 \%, 50 \%, 75 \%$ y $100 \%$, respectivamente. La figura $4 \mathrm{~b}$, muestra el comportamiento de la utilidad en este escenario.

El escenario tendencial muestra inclinación a utilidades negativas acumuladas en tres años en la explotación porcina al ser los costos de producción más elevados que los ingresos percibidos, pero no obstante se observa una recuperación lenta en la inversión a finales de la simulación.

Los diferentes escenarios permiten visualizar que se presentan recuperaciones en las ganancias por el decremento en el número de cerdas reproductoras y en el incremento en el porcentaje de lechones vendidos. Lo que permite concluir que al cebar lechones, mayor es el porcentaje de pérdida. Según los datos obtenidos por los escenarios establecidos, se obtiene una mejor rentabilidad con la comercialización de lechones obtenidos en la granja. El principal obstáculo que se presenta en la inclinación por la comercialización de lechones, es la búsqueda de compradores que se encuentren interesados en invertir en un medio que presenta notables pérdidas a las personas que se enfocan en cebar cerdos y que de acuerdo con la simulación, no se presentan buenas panorámicas para ellos. 


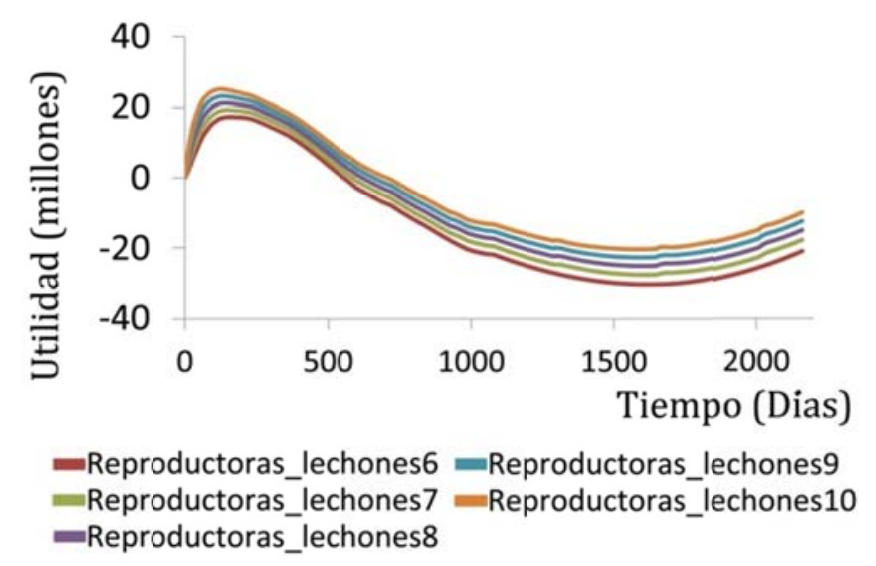

a)

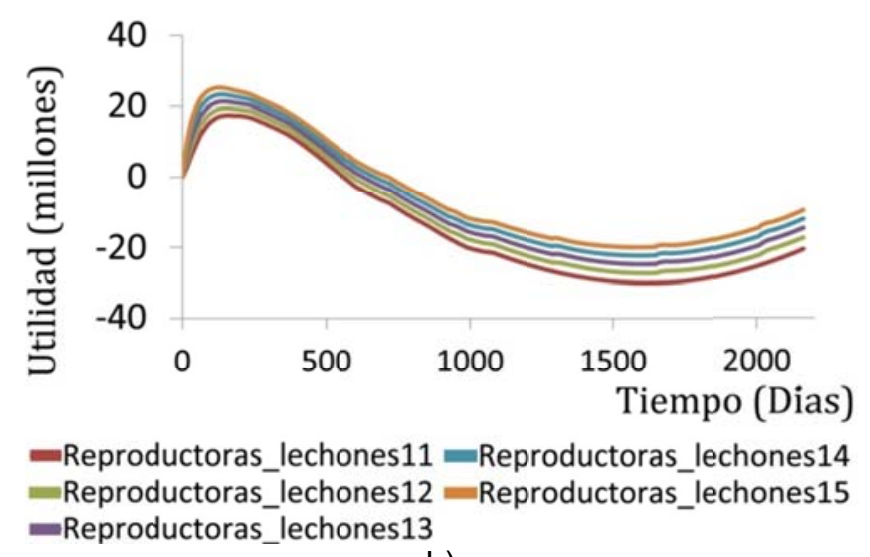

b)

Fig. 4: a) Escenario 12 madres reproductoras (utilidad en pesos Colombianos), b) Escenario 8 madres reproductoras.

No se plantean nuevos escenarios en otras variables ya que son estas dos variables las encargadas de presentar mayor influencia en el medio por ser las que en última instancia determinan la población de cerdos en la granja. Parámetros como los tiempos de retardo en las diferentes etapas de la porcicultura no se pueden afectar ya que son establecidos como fijos e inalterables bajo condiciones normales.

Los diferentes escenarios permiten visualizar que se presentan notables recuperaciones en las ganancias por el decremento en el número de cerdas reproductoras y en el incremento en el porcentaje de lechones vendidos. Lo que permite concluir que al cebar lechones, mayor es el porcentaje de pérdida.

\section{CONCLUSIONES}

De los resultados descritos, se puede concluir que ejercer la porcicultura en las fases de cría y ceba, es poco rentable para el porcicultor. El porcicultor tradicional deberá revaluar su estructura de comercialización al centrarse en la venta del cerdo en pie si pretende percibir utilidades positivas, ya sea por medio de la incursión en la comercialización de la carne de cerdo o a través de asociaciones o de alianzas con expendedores de carne.

La gran dependencia de la materia prima no le permite al porcicultor ser más competitivo en el medio, debido a que los altos costos de los insumos, terminan por afectar de forma negativa la utilidad. El modelo propuesto constituye una herramienta para analizar estrategias de mercadeo para ell porcicultor, permitiendo analizar los efectos en los ingresos y los costos de cada una de las variables involucradas en este sector económico.

Se deja a consideración de trabajos futuros la adición de la fase de comercialización de la carne de cerdo por parte de los porcicultores para así analizar los precios que tendrían la carne de cerdo y el cerdo en pie. Además, realizar un análisis de la dinámica de los cerdos reproductores que permita gestionar un comparativo entre cual forma de reproducción utilizar, si la monta natural o la inseminación artificial y decidir cuál es la mejor opción en cuestión de control microbiano, cantidad de lechones por parto, avance de los cerdos en las diferentes etapas y costos causados. 


\section{REFERENCIAS}

Cuartas B; Mosquera M. y Ladis Y., Simulador para el Aprendizaje de Toma de Decisiones en Mercadeo en el Sector Lácteos. Memorias del II Congreso Colombiano de Dinámica de Sistemas, pp. 428-439, Santa Marta, Colombia 19-21 de Agosto (2004).

Camargo, A. y Garcia, R., Evaluación de dos Modelos de Reducción de Escala en la Generación de Escenarios de Cambio Climático en el Valle de Mexicali en México. Inf. tecnol. 23(3), 11-20 (2012).

Capelo, C. y Dias, J. F., A system dynamics-based simulation experiment for testing mental model and performance effects of using the balanced scorecard. System Dynamics Review, 25(1), 1-34 (2009).

Espinal C; Martínez J. y Acevedo X., La cadena de cereales, alimentos balanceados para animales, avicultura y porcicultura en Colombia. Ministerio de Agricultura y Desarrollo Rural Observatorio Agrocadenas Colombia. Documento de trabajo. No. 51, Bogotá (2005).

Giraldo A. y Marín D., La Investigación y Desarrollo en el Sector Floricultor: Modelamiento y Análisis. II Congreso Colombiano de Dinámica de Sistemas, pp. 1-18, Santa Marta, Colombia 19-21 de Agosto (2005).

Kunc, M., Teaching strategic thinking using system dynamics: lessons from a strategic development course. System Dynamics review, 28(1), 28-45 (2012).

Padilla M., Manual de porcicultura. Ministerio de Agricultura y Ganadería. ISBN 978-9968-877-24-4, pp. 5273, San josé, Costa Rica septiembre (2010).

Paich, M., Peck, C. y Valant, J., Pharmaceutical market dynamics and strategic planning: a system dynamics perspective. Syst. Dyn. Rev., 27(1), 47-63 (2011).

Porcicol., Explotación porcícola en Colombia. Asociación colombiana de porcicultores, ISSN 0122-4220, 21(128), 25-32 (2009).

Restrepo J.; Rave J. y Peña G., Planteamiento de políticas para la solución de problemas en Pymes del sector de productos cárnicos. III Congreso Latinoamericano y III Encuentro Colombiano de Dinámica de Sistemas, Cartagena, Colombia, pp. 117-124 (2005).

Thompson J., Simulation: A Modeler's Approach. Ed. 5, pp. 328. Wiley \& Sons. England (2009). 